\title{
Automatic cutter selection based on product description and machining knowledge
}

\author{
M. Matsuda* and F. Kimura** \\ *Kanagawa Institute of Technology \\ 1030 Shimo-ogino, Atsugi-shi, Kanagawa 243-0292, Japan \\ telephone:+81-462-91-3213, fax:+81-462-42-8490 \\ e-mail: matsuda@ic.kanagawa-it.ac.jp \\ **The University of Tokyo \\ 7-3-1 Hongo, Bunkyo-ku, Tokyo 113-0033, Japan \\ telephone:+81-3-3812-2111, fax:+81-3-3812-8849 \\ e-mail: kimura@cim.pe.u-tokyo.ac.jp
}

\begin{abstract}
This paper tries to provide a methodology for a total machining system which automatically processes cutting order planning, cutter selection and generation of cutting data including generation of the cutter path based on the product model. Such an automatic machining operation planning system is presented by deriving machining features from the product description. A milling data generation system is shown as an example of automatic operation planing by milling feature derivation. In this derivation process, machining knowledge about cutters and know-how rules are used effectively. Using cutters from rough cutting to finish cutting, NC cutter paths are determined based on the milling features.
\end{abstract}

Keywords

Machining feature, product model, operation planning, manufacturing software, automatic machining, communication between design and machining, cutter selection 


\section{INTRODUCTION}

At present, if the product designer uses a CAD system, a human operator is required to input the data about machining to the CAM system. Usually the final shape data of the product which is output from a CAD system automatically becomes the input to a CAM system. Furthermore, a CAM system requires not only shape data but also designations for the machining area, data for cutters used and so on. Even if a product model generated at the design stage is available, an operator is still required to input data about machining. This means that a production engineer is required to plan machining operations and to select cutters. To realise a total machining system, planning and selection should be processed automatically.

At the product design stage, the product model is described using design features. When this product model with design features is automatically transformed to the product model with machining features, a total automatic machining system from product design to actual machining can be realised (Matsuda, 1991). This transformation mechanism showed that description of machining features were derived by applying machining knowledge, such as usable machines and cutters, to description of design features (Matsuda, 1995) (Matsuda, 1996). A general methodology for handling the product model is provided by expansion of this mechanism (Matsuda, 1997).

This paper tries to provide a methodology for automatic machining operation planning by deriving machining features from the product description using machining knowledge. A milling data generation system is shown as an example of automatic cutter selection, cutting order planning and cutting data generation by milling feature derivation.

\section{PRODUCT MODEL FOR MACHINING}

When a CAD system is used, the product model created at the design stage should be given as input data to the machining data generation system such as a CAM system. Usually, in the product model, there is information about the overall product shape, product size, material, work-piece shape and size, finishing accuracy, machining method, and so on. The product designer usually describes the product using design features, and most of the above information is described as attributes of design features or as relations between design features. However, most machining data generation systems accept only machining features in product descriptions. The automation of machining requires that the construction of a product model focuses on machining features. Here, the term "machining feature" means a volume machined by a single cutter, such as a drilled hole or milled area. On the other hand, "design feature" means a unit to achieve some function, such as transmission of motion or transformation of motion parts fixturing.

At the machining operation planning stage, it is necessary to focus on machining features. The product description used at the machining preparation stage is a 


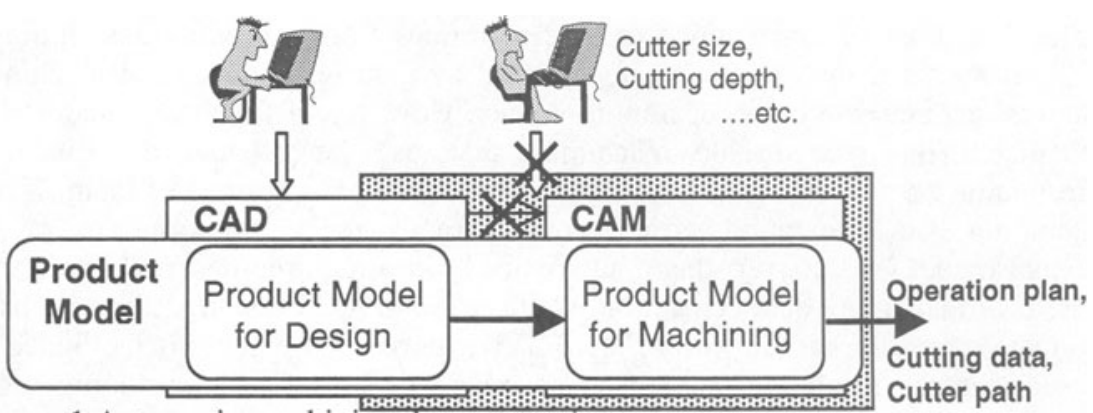

Figure 1 Automatic machining data generation.

Machining method, Using machine and cutters, Machining order, ....etc.

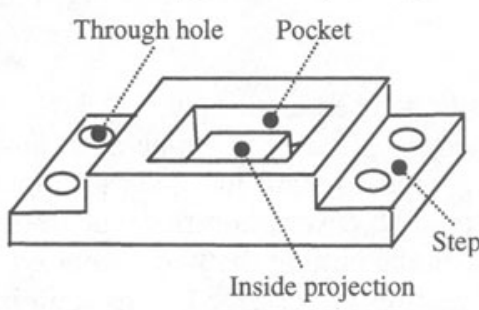

Product Description with Design Feature

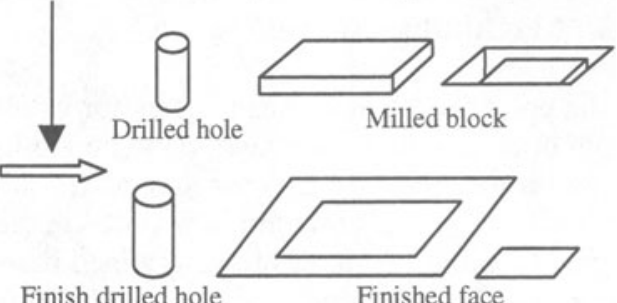

Finish drilled hole
Finished face

Product Description with

Machining Feature

Figure 2 Derivation of the machining feature descriptions.

product model that has explicit descriptions of adequate machining features. Usually, a computer system generates a cutting operation plan, the order of operation, cutting condition and control commands by using the above mentioned machining feature descriptions. In order to develop an automatic machining data generation system without any input about machining by the human operator, a machining feature generation process from product model for design should be included in the system as shown in Figure 1.

\section{AUTOMATIC MACHINING DATA GENERATION}

\subsection{Derivation of machining feature}

A product description created at the product design stage using design features implicitly includes machining features. However, explicit descriptions of machining features are required. In this case, the process of machining feature derivation becomes necessary. The derivation process derives explicit descriptions of machining features from the description of design features. Machining data which controls machines and produces actual products, is generated based on these machining features. 
The derivation of machining feature descriptions from a product description is straightforward if the design features created by a designer match the machining features recognisable by an operation planner. However, if the design features do not match the recognisable machining features, the product data includes information on machining features but not an explicit descriptions of them. If the machining data generation system could directly receive descriptions of the product model, and convert them automatically into required descriptions, a total system of machining data generation would be possible. These transformations of product description are shown in Figure 2. The derivation process is facilitated by considering the machining method using machine and cutters, machining order, and so on. The derivation process for machining features is, at same time, the process for cutter selection and planing of the cutting order.

\subsection{Machining knowledge}

In the operation planning, candidates for cutters to be used, cutter path, pick feeds, depth of cut, cutting speed and feed rate are listed for possible machine candidates based on machining features, required finishing accuracy and the material of the work-piece. This information is included in the product description from the design stage. Machining features are determined based on the cutting method, cutter type, cutter size, cutter shape, and so on. This information is described by machining knowledge. Furthermore, the selection from the possible candidates is done through consideration of cutting time, cost and so on. The method for calculating cutting time or cost is also derived from machining knowledge. Table 1 shows the classification of planning items and machining knowledge at the operation planning stage. Machining knowledge is classified into facility knowledge and process knowledge. Process knowledge includes decision rules also.

Table 1 Planning items for cutting and machining knowledge used.

\begin{tabular}{|c|c|c|}
\hline \multirow[b]{2}{*}{ Planning Item } & \multicolumn{2}{|c|}{ Machining Knowledge } \\
\hline & Facility Knowledge & Process Knowledge \\
\hline $\begin{array}{l}\text { Operation Plan } \\
\text { using cutter, } \\
\text { cutter size, } \\
\text { cutter path, } \\
\text { pick feed, } \\
\text { depth of cut, } \\
\text { cutting speed, } \\
\text { feed rate, } \\
\sim \text { etc. }\end{array}$ & $\begin{array}{l}\text { available cutters, } \\
\text { cutter type, } \\
\text { cutter shape, } \\
\text { cutter size, } \\
\text { cutting torque, } \\
\quad \sim \text { etc. }\end{array}$ & $\begin{array}{l}\text { cutting method, } \\
\text { cutting accuracy, } \\
\text { cutter interference, } \\
\text { cutting time, } \\
\text { cutting cost, } \\
\text { decision rules, } \\
\quad \sim \text { etc. }\end{array}$ \\
\hline
\end{tabular}




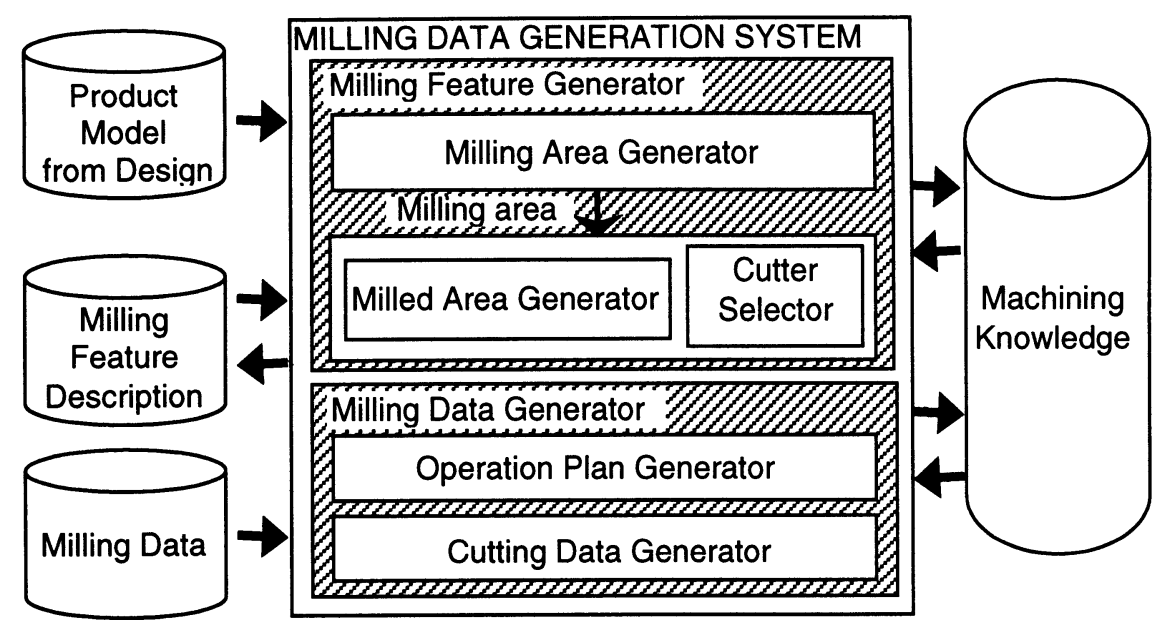

Figure 3 Structure of the milling data generation system.

\section{MILLING DATA GENERATION SYSTEM}

\subsection{Configuration of the system}

The milling data generation system receives product description from the product design stage. This system produces an operation plan for cutting as well as for milling NC data, based on the derived milling features. A milling feature is one type of machining feature. This system considers milling of a cavity for a die or mold. A cavity is one kind of a pocket. This system treats a pocket with a complicated $21 / 2$ dimensional shape and with round edge, chamfer edge and tapered face. A 2 1/2 dimensional shape means the combination of several volumes that are derived by sweeping a 2 dimensional figure. This system produces a milling operation plan from rough cutting to finish cutting. It lays out the shape of a formed cutter for finish cutting, and generates the corresponding milling data. In the rough cutting process, straight endmills are used. In order to realise high cutting efficiency, cutters with diameters as large as possible are used for rough cutting. At the intermediate cutting stage, bottom faces are finished using straight endmills. At the finish cutting stage, side faces are finished using formed cutters.

\subsection{Structure of the system}

The structure of this system is shown in Figure 3 (Matsuda, 1997). This system consists of two parts. One is the milling feature generator, and the other is the milling data generator. The product description which is obtained at the product design stage, provides inputs to the milling feature generator. In the milling feature generator, the milled cross section as milling area is determined based on the 
product shape. This is followed by the milling feature generation and cutter selection, based on the milled cross section and making use of the milling

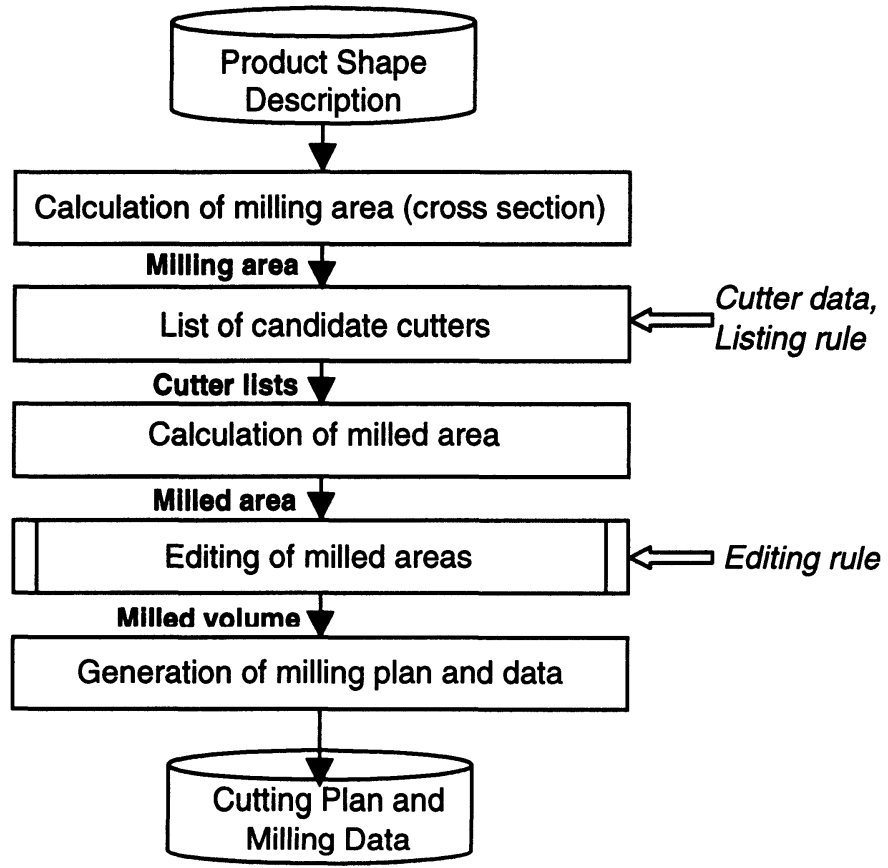

Figure 4 Process flow for milling feature generation.

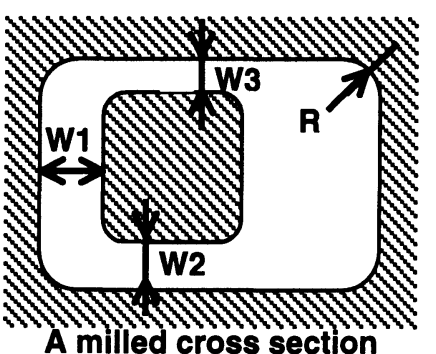

Candidate diameter, using straight endmill at rough cutting (D0, D1, D2, D3, D4)

here, $\mathrm{D} 0>\mathrm{D} 1>\mathrm{D} 2>\mathrm{D} 3>\mathrm{D} 4$

DO:biggest cutter's diameter

$D 1=<W 1-$ milling allowance

$\mathrm{D} 2=<\mathrm{W} 2-$ milling allowance

$\mathrm{D} 3=<2 R-$ milling allowance

D4 $=<$ W 3 - millina allowance

Figure 5 Selection of candidate cutter for rough cutting.

knowledge. Finally, the milling feature description is provided. The milling knowledge consists of the milling machine knowledge and milling process knowledge. The milling knowledge is a description of available cutters and knowhow rules for cutting. The milling data generator produces a cutting operation plan 
and NC data based on generated milling features through the use of the milling knowledge.

\section{CUTTER SELECTION PROCESS}

\subsection{Milling feature generation for cutter selection}

The process flow from milling feature generation to cutter selection and milling data generation is shown in Figure 4. Each step proceeds as follows:

(1) The milled cross section at each bottom face depth is computed. Each milled cross section is considered independently as a milling area.

(2) For one milling area, the candidate list of possible cutters is chosen in the list of cutter diameters of a straight endmill for rough cutting as shown in Figure 5. This process is based on the corner size, distance between island areas, and the diameter of the biggest available cutter. The cutter diameter is selected by choosing the biggest available tool on the list with a diameter that does not exceed the distance between island areas and the corner size, and also considers the milling allowance for the next cutting stage. List of available cutters and listing rules are provided by the milling knowledge. The final candidate list of applied cutter diameters consists in an ordering of cutters according to their diameters from largest to smallest, since the largest possible cutter would provide optimum milling efficiency.

(3) For each milling area, the lists of milled areas are calculated by applying each cutter on the corresponding candidate list as shown in Figure 6. The milled area is the machinable 2 dimensional figure cut by the diameter of the candidate cutter. The result of this stage is a list of resulting milled areas, and consists of a list of applied cutters, ordered according to their diameters. The milled areas are edited according to editing rules in order to obtain milled volumes. In each list of milled areas, the milled area for the bigger cutter is subtracted from the milled area for the smaller cutter. The result of the subtraction provides the actual milled area for the smaller cutter, because actual cutting starts with the bigger cutter. The result is registered as the correct milled area for the smaller cutter on the list of milled areas. However, if the result of the subtraction is smaller than the threshold area, the milled area for the smaller cutter is eliminated from the list. The threshold value is determined, based on the milling knowledge. Milled areas on all lists are divided into groups according to cutter diameter. The volume cut by a single cutter is obtained by sweeping the milled areas for one cutter, that is, one group. This milled volume corresponds to one milling feature.

(4) Milling feature descriptions are derived from to milled volumes. Milled areas which are obtained from the above step are further edited in order to get final milled volumes corresponding to each cutter used at the rough cutting, intermediate cutting and finish cutting stages. At same time this step is the selection stage for the cutters used. The details of this step is given in the next section. 
(5) Milling operation plans including lists of cutters used and cutter paths from rough cutting to finish cutting are generated, based on the milling features made up of the milled volumes corresponding to each cutter.
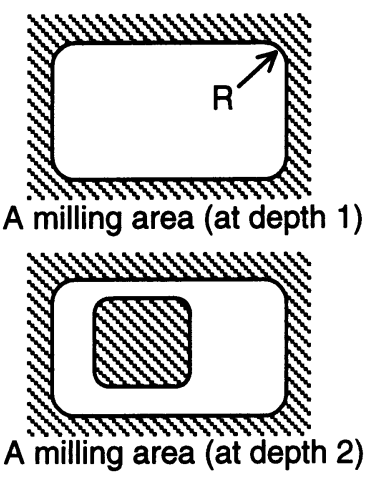

(1) Milling areas

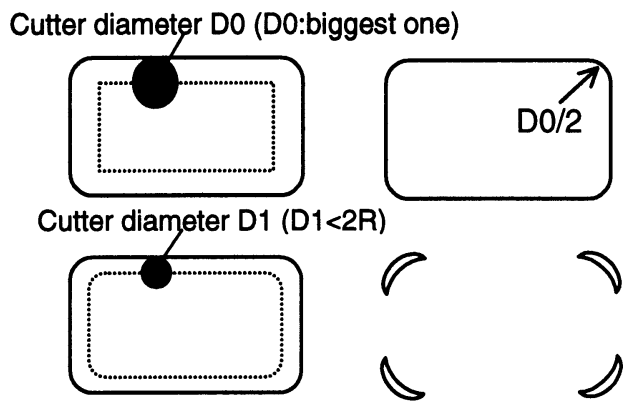

(2) Calculation of milled areas (at depth 1)
(3) Milled areas (at depth 1)

Figure 6 Calculation of milled area from milling area.

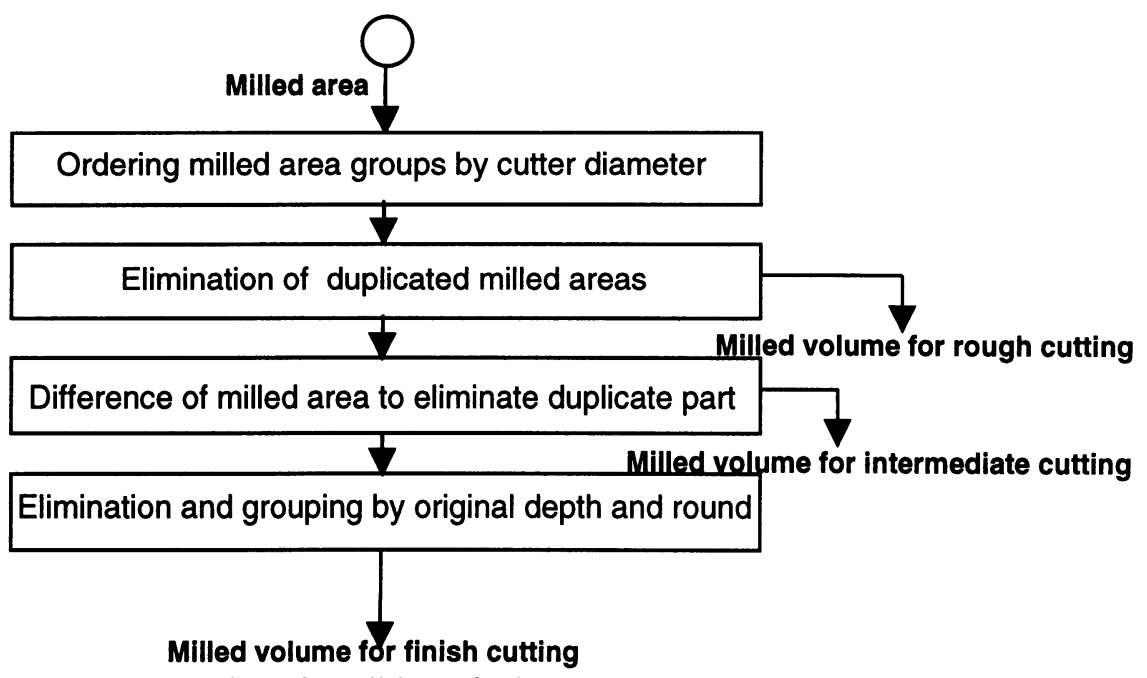

Figure 7 Process flow for editing of milled areas.

\subsection{Cutter selection process}

The editing process flow for cutter selection based on grouping of generated milled areas is shown in Figure 7. The applied editing rules form one kind of milling knowledge. Each step proceeds as following. 
(1) The milled area groups which were obtained according to cutter diameter from previous step are ordered by cutter diameter size, largest to smallest.

(2) In one group, milled areas are ordered by depth, shallow to deep, and duplicated milled areas at each depth are eliminated. Here, groups of milled areas for rough cutting are determined. One group of milled areas equals a milling feature. Here, cutters for rough cutting are selected.

(3) If two milled areas in one group have a duplicated part as seen from the top view, there is no bottom face on the duplicated part. In order to eliminate the duplicated part, the difference between the two milled areas is computed. The milled area groups which are obtained after computing for all milling duplications correspond to the milling features for intermediate cutting.

(4) The depth of each milled area for intermediate cutting corresponds to the depth of the edges of the original faces. If there is no correspondence, that milled area is eliminated. One group is divided into smaller groups by the original edge attribute of the milled area, such as "round." Formed cutter designs are determined depending on requirements of these small groups. Each of these small groups corresponds to a milling feature for finish cutting.

\section{CONCLUSIONS}

In this paper, a total machining system is proposed, which uses inputs of a product model with design features and generates machining data, without requiring input from a human operator. In other words, a methodology for generating machining features using the product description from the design stage and machining knowledge was presented. Automatic cutter selection and milling data generation was obtained by applying this methodology. This milling data generation system also showed that a total machining operation planning system from product design to machine control data generation could be structured without a human operator.

\section{ACKNOWLEDGEMENTS}

The authors are grateful to Dr. Udo Graefe of National Research Council of Canada for his helpful advice. And the authors would like to express our thanks to MATRA Data Vision Co. for their useful assistance.

\section{REFERENCES}

Matsuda, M. and Kimura, F. (1991) Extraction of machining feature for milling data generation, in Computer Application in Production and Engineering, IFIP CAPE'91, 353-360.

Matsuda, M. and Kimura, F. (1995) Modeling of virtual manufacturing devices for machining data generation, in Computer Application in Production and Engineering, IFIP CAPE'95, 407-414. 
Matsuda, M. and Kimura, F. (1996) Generation of milling data in a virtual manufacturing framework, in Design of Information Infrastructure Systems for Manufacturing, IFIP DIISM'96, 277-288.

Matsuda, M. and Kimura, F. (1997) Machining feature generation based on a facility model and manufacturing knowledge, in Computer Application in Production and Engineering, IFIP CAPE'97, 268-277.

\section{BIOGRAPHY}

M.Matsuda is an associate professor in the Department of Information and Computer Sciences of Kanagawa Institute of Technology. She received a Dr. Eng. degree in precision machinery engineering from the University of Tokyo in 1989. She was an associate professor in the Department of Computer Science of Sanno Institute of Management from 1991 to March 1997. She is a member of Japan Society of Precision Engineering and Information Processing Society of Japan.

F.Kimura is a professor in the Department of Precision Machinery Engineering of the University of Tokyo. He received a Dr. Eng. Sci. degree in aeronautics from the University of Tokyo in 1974. He was a research associate at the Electroacoustics Laboratory of the Ministry of International Trade and Industry from 1974 to 1979 . He then moved to the University of Tokyo, and was an associate professor from 1979 to 1987 . He is a member of IFIP WG5.2 and WG5.3, and a member of CIRP. 